\title{
Epidemiological and clinical aspects of skin diseases observed in workers handling cement in Burkina Faso
}

\author{
Issouf Konaté ${ }^{1,2,3}$, Nina Nessine Korsaga/Somét,5, Arsène Hema ${ }^{3}$, \\ Samira Véronique Sanfo ${ }^{1}$, Boukary Diallo ${ }^{1,3}$, Jean Baptiste Andonaba ${ }^{1,3}$, \\ Fatou Barro/Traoré4,5, Pascal Niamba ${ }^{4,5}$, Adama Traoré 4,5
}

${ }^{1}$ High Institute of Health Sciences; Nazi BONI University, Bobo-Dioulasso, Burkina Faso; ${ }^{2}$ Centre Muraz, National Institute of Public Health, Bobo-Dioulasso, Burkina Faso; ${ }^{3}$ Souro Sanou University Hospital Center, Bobo-Dioulasso, Burkina Faso; ${ }^{4}$ Faculty of Health Sciences, Joseph KI-ZERBO University, Ouagadougou, Burkina Faso; ${ }^{5}$ Service of Dermatology and Venereology, Yalgado Ouedraogo University Hospital Center, Ouagadougou, Burkina Faso

Corresponding author: Dr. Issouf Konaté, E-mail: kletio@yahoo.fr

\begin{abstract}
Background: The building and public works sector is booming in recent years in Burkina Faso and exposes workers to different materials including cement. The aim of this study was to describe the epidemiological and clinical characteristics of skin diseases observed among cement workers in Burkina Faso. Patients and Methods: We conducted a cross-sectional descriptive study, from April to June 2015, on 22 sites in the cities of Ouagadougou and Bobo Dioulasso. The study concerned workers on construction sites, handling cement and giving their agreement. A standardized survey form was designed for socio-demographic and dermatological examination data collection. Results: The study included 300 workers, all male. The median age was 29 years old. The extreme ages were 16 and 66 years old. The workers were masons (39.7\%) and apprentice masons (34.7\%). Two hundred and sixteen workers had a history of cement dermatitis and 56 had already observed a temporary cessation of work. Of the 300 workers, 265 had at least one dermatoses. The dermatoses prevalence was $88.3 \%$. We recorded a prevalence of $12 \%$ cement contact dermatitis including $8.7 \%$ irritation contact dermatitis and $3.3 \%$ allergic contact dermatitis. We observed a total of 369 dermatoses including 237 keratoderma and 69 mycotic dermatoses. Conclusion: Dermatosis is common among workers who handle cement. They are dominated by keratoderma, mycotic dermatosis and cement contact dermatitis. Etiological investigations will be necessary in our context.
\end{abstract}

Key words: Skin diseases; Cement workers; Burkina Faso

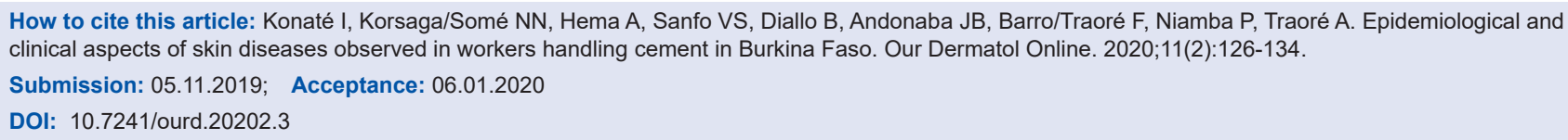




\title{
Aspects epidemiologiques et cliniques des dermatoses observees chez les ouvriers manipulant le ciment au Burkina Faso
}

\author{
Issouf Konaté ${ }^{1,2,3}$, Nina Nessine Korsaga/Somét,5, Arsène Hema ${ }^{3}$, \\ Samira Véronique Sanfo', Boukary Diallo ${ }^{1,3}$, Jean Baptiste Andonaba ${ }^{1,3}$, \\ Fatou Barro/Traoré4,5, Pascal Niamba ${ }^{4,5}$, Adama Traoré 4,5
}

\begin{abstract}
${ }^{1}$ High Institute of Health Sciences; Nazi BONI University, Bobo-Dioulasso, Burkina Faso; ${ }^{2}$ Centre Muraz, National Institute of Public Health, Bobo-Dioulasso, Burkina Faso; ${ }^{3}$ Souro Sanou University Hospital Center, Bobo-Dioulasso, Burkina Faso; ${ }^{4}$ Faculty of Health Sciences, Joseph KI-ZERBO University, Ouagadougou, Burkina Faso; ${ }^{5}$ Service of Dermatology and Venereology, Yalgado Ouedraogo University Hospital Center, Ouagadougou, Burkina Faso
\end{abstract}

Corresponding author: Dr. Issouf Konaté, E-mail: kletio@yahoo.fr

\begin{abstract}
RESUME
Background: Le secteur du bâtiment et des travaux publics connaît un essor important ces dernières années au Burkina Faso et expose les ouvriers à différents matériaux dont le ciment. Le but de cette étude était de décrire les caractéristiques épidémiologiques et cliniques des dermatoses observées chez les ouvriers qui manipulent le ciment au Burkina Faso. Patients et Méthodes: Nous avons mené une étude descriptive transversale qui s'est déroulée du 08 Avril au ler Juin 2015, sur 22 chantiers des villes de Ouagadougou et de Bobo Dioulasso. Létude concernait les ouvriers se trouvant sur les chantiers de construction, manipulant le ciment et ayant donné leur accord. Une fiche d'enquête standardisée était conçue pour la collecte des données socio démographiques, et des données de l'examen dermatologique. Résultats: Létude a inclus 300 ouvriers, tous de sexe masculin. L'âge médian des ouvriers était de 29 ans. Les âges extrêmes étaient de 16 et 66 ans. Les ouvriers enquêtés étaient maçons $(39,7 \%)$ et apprenti-maçons $(34,7 \%)$. Deux cent seize ouvriers avaient un antécédent de dermatose due au ciment et 56 avaient déjà observé un arrêt temporaire du travail. Parmi les 300 ouvriers, 265 présentaient au moins une affection dermatologique, soit une prévalence des dermatoses de $88,3 \%$. Nous avons enregistré une prévalence de $12 \%$ de dermite de contact au ciment dont 8,7\% de dermites de contact d'irritation et 3,3\% de dermites de contact allergique. Nous avons observé en tout 369 dermatoses dont 237 kératodermies et 69 dermatoses mycosiques. Conclusion: Les dermatoses sont fréquentes chez les ouvriers qui manipulent le ciment. Elles sont dominées par les kératodermies, les dermites de contact au ciment et les dermatoses mycosiques. Des enquêtes étiologiques seront nécessaires dans notre contexte.
\end{abstract}

Mots clés: Dermatoses; Ouvriers; Ciment; Burkina Faso

\section{INTRODUCTION}

Dans le secteur du travail, les maladies professionnelles résultent des dysfonctionnements provenant des interactions entre les facteurs humains, les facteurs techniques, l'environnement de travail et les facteurs liés à l'organisation du travail [1].

De nos jours, environ 20 à $34 \%$ des maladies professionnelles en Europe sont des dermatoses [2].
Les dermatoses professionnelles représentent plus de $10 \%$ de la pathologie cutanée en France et touchent 1 à $2 \%$ des salariés en activité [3].

Les dermatoses professionnelles relèvent de causes multiples parmi lesquelles on peut distinguer des agents infectieux, des agents physiques et des facteurs chimiques [4]. Le secteur de la construction représente un des secteurs les plus à risque de dermatoses professionnelles avec le ciment comme premier

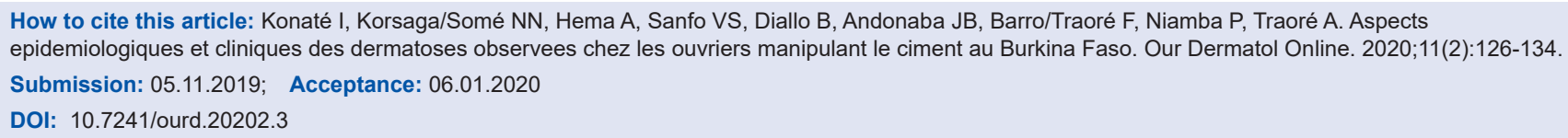


facteur causal, agissant à la fois comme irritant et allergisant [2,5-7].

Au Burkina Faso, les dermites irritatives et les dermites eczématiformes figurent sur la liste des maladies professionnelles indemnisables [1]. Le secteur du Bâtiment et des Travaux Publics (BTP) connaît un essor important ces dernières années. Il s'agit d'un secteur important pour l'économie du pays. Cette activité expose à de nombreuses poussières et à différents matériaux dont le ciment. Le contact avec le ciment reste important, surtout chez dans les organisations informelles, dans les petites entreprises où le travail se réalise dans des conditions difficiles [8]. Le but de la présente étude était de décrire les caractéristiques épidémiologiques et cliniques des dermatoses observées chez les ouvriers usagers du ciment sur les chantiers du BTP à Ouagadougou et à Bobo-Dioulasso.

\section{MATERIELS ET METHODES}

\section{Type, Période et Cadre de L'étude}

Il s'est agi d'une étude descriptive transversale qui s'est déroulée du 08 Avril au ler Juin 2015. Elle a eu pour cadre les chantiers du BTP dans les deux principales villes du Burkina Faso qui sont Ouagadougou la capitale politique et Bobo Dioulasso la capitale économique.

\section{Echantillonnage et Méthode de Sélection Des Ouvriers}

Le calcul de la taille minimale de notre échantillon était basé sur la formule de Swartzch [9] selon laquelle $\mathrm{n}=\left[\mathrm{Z}^{2} \mathrm{p}(1-\mathrm{p}) / \mathrm{i}^{2}\right]$. Elle permet d'estimer la proportion des dermatoses chez les ouvriers du ciment avec une précision donnée. Dans cette formule: $\mathrm{n}=$ taille de l'échantillon, $\mathrm{Z}=$ Loi normale réduite. Nous avons choisi un risqué $\alpha=5 \%$, soit un intervalle de confiance de $95 \%$ donc z $\alpha=1,96$. «p» est la proportion supposée de la variable qualitative. Pour notre étude, nous avons considéré la prévalence de $12,48 \%$ des dermites de contact au ciment en nous basant sur l'étude de Kuruvila et coll. à Mangalore en Inde [10]. «i» est la précision désirée et pour notre étude, nous avons choisi $4 \%$.

La prise en compte de ces valeurs a permis de trouver $n=263$. Pour notre étude, nous avons examiné au total 300 ouvriers sur différents chantiers.
De façon raisonnée, nous nous sommes tout d'abord adressé à la Direction régionale du ministère de l'habitat de Bobo Dioulasso puis de Ouagadougou, pour cibler les chantiers du BTP. Au niveau des chantiers, nous avons proposé systématiquement à tous les ouvriers consentant de les examiner et ce jusqu'à l'obtention du nombre de sujets nécessaires.

\section{Variables Étudiées, Collecte et Analyse Des Données}

Nous nous sommes intéressés aux variables épidémiologiques (sexe, âge, religion, statut marital, niveau de scolarisation, poste de travail, ville d'exercice, durée de l'exposition au ciment, régularité de l'exposition au ciment, port d'Equipements de Protection Individuels (EPI)), et cliniques (antécédents de dermatoses liée au ciment, antécédent d'arrêt de travail pour dermatose liée au ciment, issue de la dermatose liée au ciment après l'arrêt de travail, présence d'atopie personnel et/ou familiale, consommation d'alcool, consommation de tabac, présence de dermatoses à l'interrogatoire et à l'examen physique et type de dermatose).

La collecte des données était faite à partir d'une fiche d'enquête standardisée, renseignée par un entretien direct, en face à face avec l'ouvrier, et complétée par les données de l'examen physique.

Les données étaient analysées avec le logiciel statal2. Pour les facteurs associés, nous avons utilisé un seuil de signification de $5 \%$. En analyse uni variée, nous avons utilisé le test du Chi carré de Pearson. En multivariée, nous avons utilisé une régression logistique. Le seuil d'inclusion des variables dans le model était de $30 \%$.

\section{Aspects Éthiques}

Nous avons obtenu les autorisations des responsables des entreprises qui ont servi de site d'étude. Nous avons recueilli le consentement des ouvriers avant leur inclusion. Lanonymat des ouvriers a été respecté. Lorsqu'une dermatose était retrouvée chez un ouvrier, des conseils étaient prodigués ainsi qu'une prescription médicale gratuite. La retro information sur les recommandations validées étaient faite aux entreprises. 


\section{RÉSULTATS}

\section{Aspects Épidémiologiques}

\section{Fréquence globale des dermatoses parmi l'ensemble} des ouvriers

Létude a concerné au total 300 ouvriers sur 22 chantiers du BTP répartis dans le tableau I. Parmi les 300 ouvriers inclus, 265 ouvriers présentaient au moins une affection dermatologique, soit une prévalence des dermatoses de $88,33 \%, \mathrm{IC}_{95 \%}=[84,69-91,96]$. Parmi ces 265 ouvriers, $216(81,15 \%)$ répondaient n’avoir pas de dermatoses à l'interrogatoire.

Caractéristiques sociodémographiques et professionnelles des ouvriers enquêtés

Les ouvriers étaient tous de sexe masculin. L'âge médian des ouvriers était de 29 ans. Les âges extrêmes étaient de 16 et 66 ans. Ils étaient musulmans dans 52,33\% des cas $(n=157)$. La moitié des ouvriers vivaient en couple, $67 \%$ avaient un niveau de scolarisation qui ne dépassait pas le primaire.

Selon le poste de travail, les maçons et les apprentimaçons avaient des fréquences respectives de 39,67\% et 34,67\%. Les autres catégories professionnelles étaient représentées par des magasiniers de ciment, des électriciens, des techniciens du bâtiment avec un effectif égal à 3 pour chacune d'elles; des carreleurs, des stagiaires en génie civil, des livreurs de ciment, des plombiers, des tacherons avec un effectif égal à 2 pour chacune d'elles; on y trouvait également un peintre et un contrôleur. Deux cent quarante-six ouvriers $(82 \%)$ étaient recrutés à Ouagadougou.

Selon le degré d'exposition au ciment, 147 ouvriers (49\%) étaient exposés au ciment depuis plus de 5 ans, $151(50,3 \%)$ avaient un contact régulier avec le ciment et $191(63,7 \%)$ affirmaient ne jamais porter d'EPI.

\section{Aspects cliniques}

Selon les antécédents dermatologiques et allergiques, parmi les 300 ouvriers qui manipulaient le ciment, $216(72 \%)$ ouvriers avaient déjà présenté au cours de leur exercice au moins un épisode de dermatose due au ciment et $56(18,67 \%)$ avaient déjà observé un arrêt temporaire de travail allant de 24 heures à 03 mois, pour cause d'affections dermatologiques dues au ciment. Tous les ouvriers qui avaient déjà observé un arrêt de travail pour dermatose due au ciment avaient constaté une amélioration de leur dermatose pendant l'arrêt du contact avec le ciment.

La fréquence des ouvriers qui avaient une atopie personnelle uniquement était de $16 \%(n=48)$, celle des ouvriers ayant une atopie familiale uniquement était de $12,33 \%(n=37)$. La fréquence des ouvriers qui avaient une atopie personnelle et familiale était de

\begin{tabular}{|c|c|c|c|}
\hline $\mathrm{N}^{\circ}$ ordre & Description de l'ouvrage en cours & Ville & Nombre d'ouvriers \\
\hline 1 & Construction d'une infirmerie pour le centre Muraz & Bobo* & 12 \\
\hline 2 & Travaux d'aménagement de la plateforme du village artisanal de bobo Dioulasso & bobo & 14 \\
\hline 3 & Travaux de voirie et d'assainissement de la zone SONATUR section 447 & bobo & 16 \\
\hline 4 & Construction d'une agence de Coris Banque à Gounghin & Ouaga* $^{* *}$ & 21 \\
\hline 5 & Agence de placement d'ouvriers du bâtiment & Ouaga & 10 \\
\hline 6 & Construction d'un bâtiment $\mathrm{R}+1$ à usage de magasins & Ouaga & 14 \\
\hline 7 & Construction d'un laboratoire à l'IRSS (institut de recherche en science de la santé) & Ouaga & 9 \\
\hline 8 & Construction d'une salle de cours à l'université de Ouagadougou & Ouaga & 20 \\
\hline 9 & Construction d'un bâtiment administratif du ministère de l'enseignement de base & Ouaga & 17 \\
\hline 10 & Construction d'un immeuble $\mathrm{R}+2$ à usage de bureaux pour la primature & Ouaga & 45 \\
\hline 11 & Construction d'un bâtiment de pédagogie à l'université de Ouagadougou & Ouaga & 12 \\
\hline 12 & Travaux d'aménagement de voirie à Saaba & Ouaga & 9 \\
\hline 13 & Construction d'un amphithéâtre de 700 places à l'Institut des Sciences & Ouaga & 14 \\
\hline 14 & Construction d'un bâtiment $R+1$ à usage de magasin & Ouaga & 14 \\
\hline 15 & Construction d'un bâtiment $R+1$ à usage de bureaux & Ouaga & 7 \\
\hline 16 & Construction de l'Ambassade de l'Arabie Saoudite à Ouaga 2000 & Ouaga & 20 \\
\hline 17 & Construction d'un bâtiment administratif $\mathrm{R}+3$ avec sous terrain & Ouaga & 12 \\
\hline 18 & Construction d'un bâtiment $R+3$ à usage de bureaux & Ouaga & 6 \\
\hline 19 & Construction d'un magasin à la ZAD & Ouaga & 5 \\
\hline 20 & Construction d'un bâtiment à usage de bureau à la ZAD & Ouaga & 6 \\
\hline 21 & Construction de l'Ambassade du Mali à Ouaga 2000 & Ouaga & 15 \\
\hline 22 & Construction d'un bâtiment $R+1$ à usage de boite de nuit & Ouaga & 2 \\
\hline
\end{tabular}

$\left({ }^{\star}\right)=$ Bobo-Dioulasso et $\left(^{* \star}\right)=$ Ouagadougou 
$13 \%$ et celle des ouvriers qui ne présentaient aucune atopie était de 58,66\% $(\mathrm{n}=176)$.

Selon la consommation d'alcool et de tabac, 129 ouvriers consommaient l'alcool et 96 du tabac.

\section{Etude Des Dermatoses Observées}

\section{Répartition des différentes dermatoses selon leurs} proportions et leurs prévalences

Les kératodermies représentaient 64,23\% de l'ensemble des dermatoses. Les mains étaient atteintes dans 97,46 $\%$ des cas. Les dermites de contact au ciment avaient une prévalence de 12\% (Figs. 1-3).

Les dermatoses mycosiques étaient au nombre de 69 dont 52 cas d'intertrigo inter orteil dermatophytique et 10 cas de pityriasis versicolor. Les autres dermatoses les plus fréquentes étaient l'acné (10 cas) et la xérose

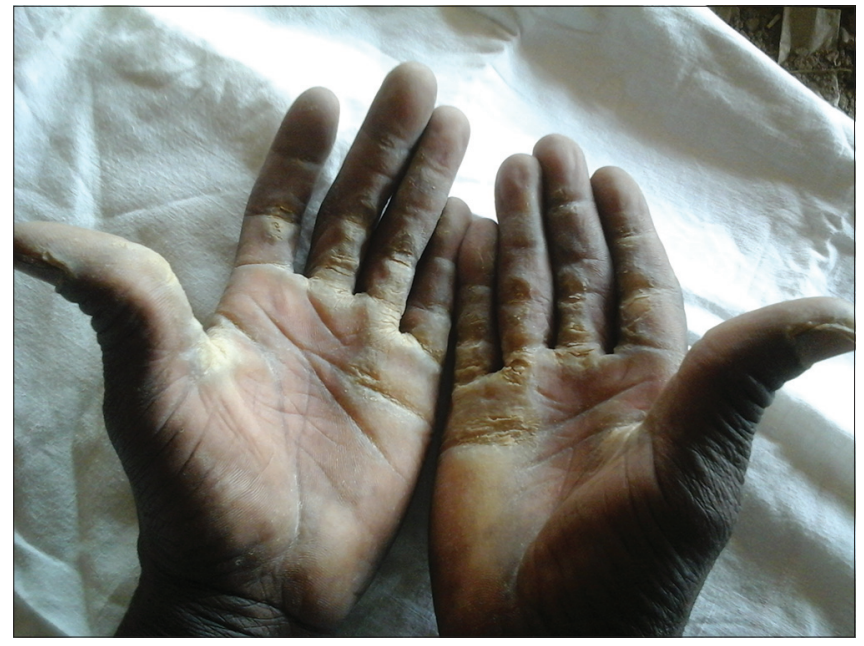

Figure 1: Keratodermie palmaire chez un maçon.

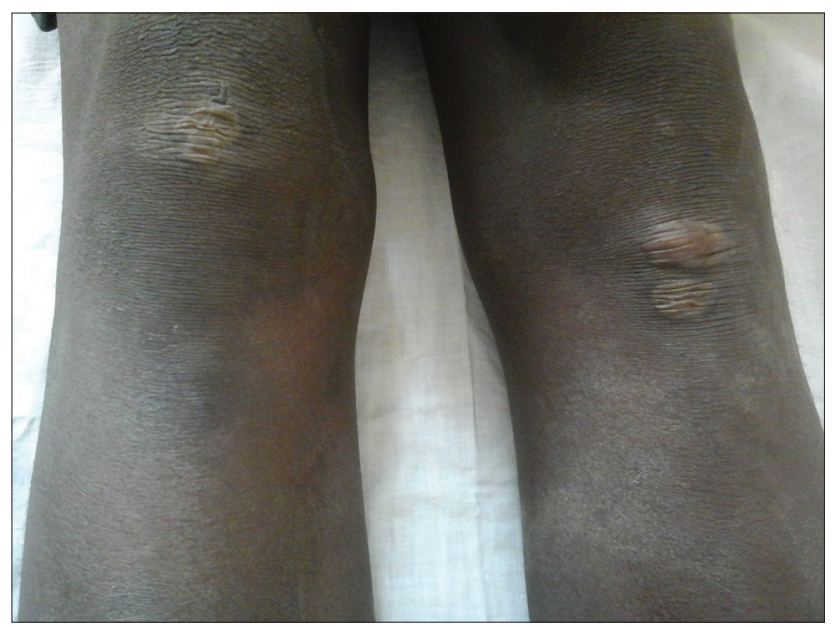

Figure 2: Dermite d'irritation des genoux (survenue au lendemain d'un crépissage à genoux).
(8 cas). La répartition des dermatoses en fonctions de leur prévalence et de leur proportion est présentée dans le tableau II.

\section{Etude des facteurs associés aux dermites de contact} La fréquence des dermites de contact était de 14,1\% et $6,9 \%$ respectivement parmi les ouvriers de moins de 35 ans et de plus de 35 ans. OR non ajusté $=0,4[0,2$ $-0,1]$. Le tableau III montre les résultats d'analyse des facteurs de risque de dermite de contact.

\section{DISCUSSION}

Nous avons mené une étude transversale descriptive parmi 300 ouvriers du BTP dans le but de décrire les caractéristiques épidémiologiques et cliniques des dermatoses observées chez les ouvriers manipulant le ciment. Labsence d'utilisation des patch-tests ne nous a pas permis d'identifier de façon formelle les allergènes en cause, mais par contre notre enquête anamnestique et l'examen clinique ont permis d'incriminer le ciment dans l'apparition de ces affections. Les autres diagnostic étaient basés sur les arguments cliniques.

Les résultats obtenus à travers cette étude ont néanmoins permis d'avoir des données de base sur la fréquence des différentes dermatoses observées dans ce groupe des manipulateurs du ciment au Burkina Faso.

\section{Des Caractéristiques Des Ouvriers}

\section{Du sexe}

Tous les ouvriers de notre enquête étaient de sexe masculin. Le programme "Build up skills" (BUS) [11] dans son rapport publié en janvier 2013 en France a révélé que seulement 1,6\% des ouvriers du secteur du BTP sont de sexe féminin. Dans l'étude de Balkrishna B. et coll. [12] en 2010 au sein des ouvriers migrants de la construction en Inde, tous les ouvriers enquêtés étaient de sexe masculin. Ces similitudes montrent véritablement que le secteur du BTP est un secteur fortement masculin probablement du fait de l'endurance physique, la rudesse du métier et les conditions hygiéniques précaires du milieu qui ne favorisent pas la féminisation de ce métier.

\section{De l'âge}

les âges extrêmes dans l'étude de Kuruvila [10] étaient de 14 et 63 ans, ce qui est similaire avec nos résultats (16 et 66 ans); prouvant encore le caractère informel de ce secteur d'activités avec la non régulation de l'âge légal de travail. C'est le constat que fait 
www.odermatol.com

Tableau II: Répartition des 369 cas de dermatoses en fonctions de leur prévalence et de leur proportion

\begin{tabular}{|c|c|c|c|}
\hline Dermatoses & Effectifs & Proportion (\%) $n=369$ & Prévalence $(\%) \mathrm{n}=300$ \\
\hline Kératodermies & 237 & 64,23 & 79,00 \\
\hline \multicolumn{4}{|l|}{ Dermatoses mycosiques } \\
\hline Intertrigo inter-orteils & 52 & 14,09 & 17,33 \\
\hline Pityriasis versicolore & 10 & 2,71 & 3,33 \\
\hline Autres dermatoses mycosiques( $\left.{ }^{*}\right)$ & 7 & 1,90 & 2,33 \\
\hline Total dermatoses mycosiques & 69 & 18,70 & NA \\
\hline \multicolumn{4}{|l|}{ Dermites de contact au ciment } \\
\hline $\mathrm{DCl}$ & 26 & 7,05 & 8,66 \\
\hline DCA & 10 & 2,71 & 3,33 \\
\hline Total dermites de contact au ciment & 36 & 9,76 & 12,00 \\
\hline Autres dermatoses $\left({ }^{* \star}\right)$ & 27 & 7,31 & NA \\
\hline Total dermatoses & 369 & 100 & 100 \\
\hline
\end{tabular}

(")=les autres dermatoses mycosiques étaient 02 cas de dermatophyties inguinales, 02 cas de dermatophyties de la peau glabre, 02 cas de dermatophytie unguéale et 01 cas d'onyxis candidosique. (")=les autres dermatoses étaient représentées par 12 cas d'acné, 8 cas de xérose, 02 cas de réactions aux sousvêtements, 01 eczéma de contact aux chaussures en cuir, 01 cas d'eczéma atopique, 01 cas de nævus, 01 cas de folliculite, et 01 cas de chéloïde. NA=Non approprié car un même ouvrier peut être éligible pour plusieurs autres dermatoses

Tableau III: Analyse des facteurs de risque de dermites de contact au ciment

\begin{tabular}{|c|c|c|c|c|}
\hline Caractéristiques & $\begin{array}{c}\text { Proportions des } \\
\text { dermites de contacts }\end{array}$ & $\begin{array}{l}\text { Odds ratio non ajustés } \\
\text { (analyse uni variée) }\end{array}$ & $\begin{array}{l}\text { Odds ratio ajustés } \\
\text { (analyse multivariée) }\end{array}$ & $P^{* *}$ values \\
\hline \multicolumn{5}{|l|}{ Classes d'âges } \\
\hline$[15-35$ ans $]$ & $30 / 213$ & 1,0 & 1,0 & \\
\hline Plus de 35 ans & $6 / 87$ & $0,4[0,2-0,1]$ & $0,5[0,2-1,3]$ & 0,16 \\
\hline \multicolumn{5}{|l|}{ Niveau de scolarisation } \\
\hline Aucun & $7 / 56$ & 1,0 & 1,0 & \\
\hline Primaire & $12 / 145$ & $0,6[0,2-1,7]$ & $0,7[0,2-1,9]$ & 0,49 \\
\hline Secondaire & $15 / 86$ & $1,5[0,5-3,8]$ & $1,5[0,5-4,0]$ & 0,45 \\
\hline Supérieur & $2 / 13$ & $1,3[0,2-6,9]$ & $1,1[0,2-6,5]$ & 0,92 \\
\hline \multicolumn{5}{|l|}{ Poste de travail } \\
\hline Menuisier & $3 / 29$ & 1,0 & - & \\
\hline Maçon & $19 / 119$ & $1,6[0,4-5,9]$ & - & \\
\hline Apprentis maçons & $11 / 104$ & $1,0[0,3-3,9]$ & - & \\
\hline Bétonniers & $2 / 12$ & $1,7[0,2-11,9]$ & - & \\
\hline Ferrailleurs* & $0 / 8$ & - & - & \\
\hline Briquetiers $^{*}$ & $0 / 7$ & - & - & \\
\hline Autres & $1 / 21$ & $0,4[0,1-4,5]$ & - & \\
\hline \multicolumn{5}{|c|}{ Durée du contact avec le ciment } \\
\hline [0-1 an] & $21 / 151$ & 1,0 & 1,0 & \\
\hline 1 an et plus & $15 / 149$ & $0,5[0,2-1,1]$ & $0,6[0,3-1,5]$ & 0,29 \\
\hline \multicolumn{5}{|c|}{ Régularité du contact avec le ciment } \\
\hline Plein temps & $21 / 151$ & 1,0 & 1,0 & \\
\hline Occasionnels & $15 / 149$ & $0,7[0,3-1,4]$ & $0,6[0,3-1,2]$ & 0,14 \\
\hline \multicolumn{5}{|l|}{ Utilisation d'EPI } \\
\hline Toujours & $2 / 27$ & 1,0 & 1,0 & \\
\hline Pas toujours & $34 / 273$ & $0,5[0,1-2,5]$ & $0,5[0,1-2,5]$ & 0,42 \\
\hline \multicolumn{5}{|l|}{ Consommation de tabac } \\
\hline Non & $22 / 204$ & 1,0 & - & \\
\hline Oui & $14 / 96$ & $1,4[0,7-2,9]$ & - & \\
\hline \multicolumn{5}{|l|}{ Consommation d'alcool } \\
\hline Jamais & $23 / 171$ & 1,0 & 1,0 & \\
\hline Occasionnellement & $3 / 44$ & $0,5[0,1-1,6]$ & $0,4[0,1-1,6]$ & 0,21 \\
\hline Régulièrement & $10 / 85$ & $0,8[0,4-1,9]$ & $0,9[0,4-2,1]$ & 0,81 \\
\hline \multicolumn{5}{|l|}{ Absence d'atopie } \\
\hline Non & $13 / 124$ & 1,0 & - & \\
\hline Oui & $19 / 176$ & $0,7[0,4-1,5]$ & - & \\
\hline
\end{tabular}

$\left({ }^{*}\right)$ : Exclus de l'analyse $\left({ }^{* *}\right)$ : Obtenu après ajustement sur l'âge, le niveau scolaire, la durée de contact avec le ciment, la régularité du contact avec le ciment, l'utilisation d'EPI et la consommation régulière d'alcool

également le Ministère de l'économie, des finances et de l'emploi en partenariat avec le Ministère du travail, des relations sociales et de la solidarité en France en 2008 [13].

\section{Des catégories professionnelles}

les plus représentées étaient les maçons $(39,67 \%)$ et les apprenti-maçons $(34,67 \%)$, c'est le même constat qu'a fait Kuruvila [10] dans son étude avec 51,20\% 


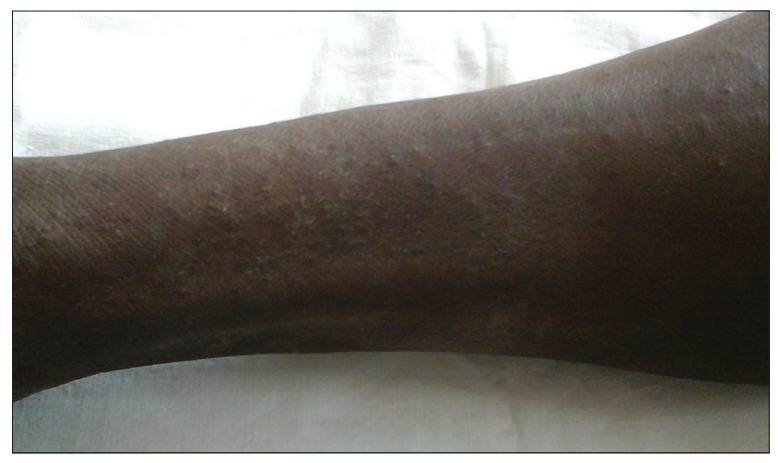

Figure 3: Dermite de contact allergique de l'avant-bras.

pour les apprenti-maçons et 32,78 \% pour les maçons. Ces résultats sont en accord avec le fait que sur les chantiers du BTP ces 2 catégories professionnelles sont les plus impliquées.

\section{Du niveau d'instruction}

seuls 10,27 \% des ouvriers étaient alphabétisés dans l'étude de Kuruvila [10] contre 81,33\% dans notre étude; cette disparité peut s'expliquer par le fait que l'étude de Kuruvila ne concernait que des migrants qui ont quitté leurs pays à la recherche de meilleures conditions de vie ce qui ne favorise pas la scolarisation; contrairement à notre étude ou la majorité étaient des nationaux.

\section{Du degré d'exposition au ciment}

Seulement $9 \%$ des ouvriers affirment toujours porter des équipements de protection avant de manipuler le ciment. Cela peut s'expliquer par le grand nombre de ces ouvriers travaillant dans le secteur informel. Ils ne bénéficient donc pas des avantages d'une couverture sociale ni des moyens de protection. Les moyens de protection sont le plus souvent acquis au prix d'économies personnelles et cela les rend parfois inadéquates.

\section{Des antécédents dermatologiques}

Les dermatoses que les ouvriers estiment être dues au ciment ont été la cause d'arrêt temporaire de travail dans les antécédents de 18,67\% des ouvriers. Ces chiffres témoignent de l'importance du handicap qu'occasionnent ces affections dermatologiques sans compter que le souci de la perte de l'emploi et de pénalités sur le salaire viennent masquer en partie la déclaration de ces affections ainsi que la décision d'arrêt temporaire de l'activité $[3,5]$.

\section{Etudes Des Fréquences}

\section{De la fréquence globale des dermatoses}

Notre étude a mis en évidence une prévalence globale des dermatoses chez les manipulateurs du ciment de $88,33 \%$. Sur les 265 ouvriers qui présentaient au moins une dermatose, 216 soit 81,15\% d'entre eux répondaient n'avoir pas de dermatoses à l'interrogatoire. Derrière ce peu d'importance accordée à la présence des dermatoses par les ouvriers, se cache parfois le souci de la perte de l'emploi et de pénalités sur le salaire $[3,5]$. Kuruvila [10] parvient à des résultats similaires avec $89,72 \%$ pour la prévalence globale des dermatoses. Cette similitude peut s'expliquer par le fait que les lieux des enquêtes (Inde et Burkina) ont des climats semblables en matière chaleur, de plus il s'agit tous deux de pays où les conditions de travail sont précaires.

Kartik R Shah [14] quant à lui retrouvait une prévalence globale des dermatoses de 47,8\%. Cette différence peut s'expliquer par une taille d'échantillon plus petite (92 ouvriers) et le mode de sélection des ouvriers sur ces chantiers s'est fait sur un mode aléatoire contre une sélection exhaustive dans notre étude.

\section{La kératodermie}

elle avait une prévalence de $79 \%$ et représentait 64,23\% de l'ensemble des dermatoses. Les mains étaient atteintes dans 97,46\% des cas. Ceci vient insister sur l'importance de l'exposition des mains dans ce travail qu'est la manipulation du ciment; cette kératodermie palmaire était provoquée par le traumatisme répété des instruments de travail tels que les pelles, brouettes, briques et probablement par l'effet chimique du ciment. Dans l'étude de Kartik R Shah,[14] la kératodermie palmaire représentait 19,6\% des dermatoses et elle était causées également par le frottement répété des outils de travail.

\section{Les intertrigos inter-orteils}

ils avaient une prévalence de $17,33 \%$ et seraient favorisés par le port prolongé des chaussures de protection mais également par l'humidité que nécessitent les lieux de travail.

\section{De la fréquence des dermites de contact}

notre étude a mis en évidence une prévalence globale des dermites de contact égal à 12\%. Kuruvila [10] parvenait à des résultats similaires $(12,48 \%)$ pour la fréquence des dermites de contact probablement du fait des resemblance socio environnementales.

Près des 3/4 des dermites de contact étaient des dermites d'irritation dans notre étude; Wang BJ [15] dans son étude chez les travailleurs du ciment à Taiwan retrouvait que les dermites de contact d'irritation représentaient près des $2 / 3$ des 
dermites de contact. Ceci corrobore l'épidémiologie de ces affections quand on sait que la DCI atteint la majorité des sujets en contact avec le produit et la DCA n'atteint que quelques sujets du fait de la sensibilisation préalable.

\section{Etude Des Facteurs Associés à La Survenue Des Dermites de Contact}

\section{L'âge}

en analyse bi variée avec un seuil de signification de $5 \%$, les ouvriers de plus de 35 ans avaient 2,5 fois moins de dermite de contact que les autres. On pourrait penser que les ouvriers jeunes seraient moins prudents et moins expérimentés en matière de prévention des dermites dues au ciment.

Après une régression logistique, en ajustant sur l'âge, le niveau scolaire, la durée de contact avec le ciment, la régularité du contact avec le ciment, l'utilisation d'EPI et la consommation régulière d'alcool aucun facteur n'était associé à la survenue des dermites de contact.

\section{Le poste de travail}

l'étude de Guo YL [16] isolait les carreleurs comme étant la catégorie professionnelle la plus à risque et dans l'étude de Kuruvila les dermites de contact étaient plus observées chez les maçons que chez les autres catégories professionnelles; ce constat peut s'expliquer par le fait que sur les chantiers qui ont fait l'objet de notre étude, les taches n'étaient pas toujours effectuées par la catégorie professionnelle indiquée, ainsi par exemple un menuisier de formation pouvait se retrouver à exécuter les taches d'un maçon ou d'un apprenti-maçon suivant l'étape du chantier et vice versa pour se trouver des revenus.

\section{L'ancienneté de contact avec le ciment}

Il n'existe pas d'association entre la présence de dermite de contact et l'ancienneté de contact avec le ciment dans notre étude. Guo YL [16] a quant à lui retrouvé une association entre l'ancienneté de travail et la survenue des dermites de contact; cette différence peut s'expliquer par le fait que malgré parfois la longue expérience de travail avec le ciment, il ne s'est pas agi en réalité d'un contact permanent et régulier, en effet près de la moitié des ouvriers travaillaient de façon occasionnelle avec le ciment (49,66\%). Les ouvriers ne sont pas employés à plein temps par des entreprises avec des périodes de flottements parfois importants entre deux chantiers contraignant l'ouvrier à se tourner vers d'autres types d'emplois.

\section{L'utilisation des EPI}

Wang BJ [15] a retrouvé que la moindre utilisation des gants était un facteur associé aux dermites de contact et Poppe H [17] retrouve dans son étude que ce sont les pauvres mesures de protection qui sont associés aux dermites de contact.

Par contre notre étude n'observe pas de lien statistique entre l'utilisation des EPI et l'actuelle présence de dermite de contact; cela pourrait traduire une utilisation non optimale des EPI.

\section{CONCLUSION}

Les dermatoses sont fréquentes, variées et le plus souvent méconnues chez les ouvriers qui manipulent le ciment. Beaucoup de ces affections sont liées à l'environnement de travail. Les dermatoses directement liées au contact avec le ciment encore appelées dermites de contact étaient fréquentes avec de multiples arrêt de travail si on prend en compte les antécédents des ouvriers. Au Burkina Faso, le secteur du BTP est un milieu où domine le secteur informel et où les conditions de travail sont parfois difficiles et précaires. Il conviendrait de mieux structurer cette catégorie professionnelle et de les doter d'un organe administratif afin de mieux prendre en compte leurs difficultés et notamment leurs multiples problèmes de santé. Notre étude n'a pas permis de mettre en évidence un lien entre les dermites de contact et des facteurs associés à leur survenue. D'autres enquêtes étiologiques sont nécessaires dans notre contexte afin d'isoler des facteurs de causalité de ces dermatoses.

\section{Statement of Human and Animal Rights}

All procedures followed were in accordance with the ethical standards of the responsible committee on human experimentation (institutional and national) and with the Helsinki Declaration of 1975, as revised in 2008 .

\section{Statement of Informed Consent}

Informed consent was obtained from all patients for being included in the study.

\section{REFERENCES}

1. Conseil des Ministres du Burkina Faso. Décret N²015-866/ PRES-TRANS/PM/MFPTSS/MS portant liste des maladies professionnelles au Burkina Faso. JO N 42 du 15 octobre 2015;p37.

2. P Frimat, $\mathrm{S}$ Fantoni-Quinton. La main du travailleur: aspect 
socio-économiques. Quelle réparation ? Quel reclassement ?. Prog Dermato-allergol Bruxelles. 2001:65-78.

3. Dezfoulian B. Dermatoses professionnelles. 2011. Disponible sur: http://www.stes-apes.med.ulg.ac.be/strav/stenseignement/ stformationcontinue/Dermatoses\%20profle.pdf. [Consulté le $21 / 10 / 2019]$

4. Géraut C, Tripodi D. Dermatoses professionnelles. Encyclopédie Médico-Chirurgicale (Elsevier, Paris), Toxicologie-Pathologie Professionnelle, 16-533-A10, Dermatologie, 98-795-A10, 2001. p. 27.

5. Crépy MN. Dermatoses professionnelles au ciment. INRS Documents pour le médecin du travail. 2001;88:419-29.

6. Macedo MS, de Avelar Alchorne AO, Costa EB, Montesano FT. Contact allergy in male construction workers in Sao Paulo, Brazil, 2000-2005. Contact Derm. 2007;56:232-4.

7. Niang SO, Cissé M, Gaye FMC, Diallo M, Boye A, Dieng MT, et al. [Occupational allergic contact dermatitis in Dakar]. Mali Med. 2007;22:34-7.

8. Frimat P. Les dermatoses professionnelles dans le bâtiment et les travaux publics. RevFrançaise d'Allergol d'Immunol Clin. 2007;47:153-7.

9. Ancelle T. Statistique, épidémiologie. 3e édition. Collection sciences fondamentales; 2011. p 80-81.

10. Kuruvila M, Dubey S, Gahalaut P. Pattern of skin diseases among migrant construction workers in Mangalore. Indian J Dermatol Venereol Leprol. 2006;72:129-32.

11. Build Up Skills. France: analyse du statu quo national: organiser la montée en compétences des ouvriers et artisans du batiment [Internet]. 2013 Jan p. 9. Available from: http://www.cercad.fr/
IMG/pdf/bus_etat_des_lieux.pdf

12. Balkrishna B. Adsul, Payal S. Laad, Prashant V. Howal, Ramesh M. Chaturvedi. Health problems among migrant construction workers: A unique public-private partnership project. Indian J Occup Envir Med. 2011;15:29-32.

13. République française, Ministère de l'économie des finances et de l'emploi, Ministère du travail, des relations sociales et de la solidarité. Les ouvriers du bâtiment et des travaux publics : des contraintes physiques et des expositions aux produits chimiques importantes, une autonomie assez élevée dans le travail. Premières synthèses, DARES. 2008; $\mathrm{N}^{\circ} 07.3: 2$.

14. Kartik R Shah, Rajnarayan R Tiwari. Occupational skin problems in construction workers. Indian J Dermatol. 2010;55:348-51.

15. Wang B-J, Wu J-D, Sheu S-C, Shih T-S, Chang H-Y, Guo Y-L, et al. Occupational hand dermatitis among cement workers in Taiwan. J Formos Med Assoc. 2011;110:775-9.

16. Guo YL, Wang BJ, Yeh KC, Wang JC, Kao HH, Wang MT, et al. Dermatoses in cement workers in southern Taiwan. Contact Derm. 1999;40:1-7.

17. Poppe H, Poppe LM, Bröcker E-B, Trautmann A. Do-it-yourself cement work: the main cause of severe irritant contact dermatitis requiring hospitalization. Contact Derm. 2013;68:111-5.

Copyright by Issouf Konaté, et al. This is an open-access article distributed under the terms of the Creative Commons Attribution License, which permits unrestricted use, distribution, and reproduction in any medium, provided the original author and source are credited.

Source of Support: Nil, Conflict of Interest: None declared. 